PROCEEDINGS OF THE

AMERICAN MATHEMATICAL SOCIETY

Volume 129, Number 4, Pages 1057-1064

S 0002-9939(00)05659-8

Article electronically published on November 8, 2000

\title{
ELEMENTS IN A COMMUTATIVE BANACH ALGEBRA DETERMINING THE NORM TOPOLOGY
}

\author{
A. R. VILLENA
}

(Communicated by Dale Alspach)

\begin{abstract}
For an element $a$ of a commutative complex Banach algebra $(A,\|\cdot\|)$ we investigate the following property: every complete norm $|\cdot|$ on $A$ making the multiplication by $a$ from $(A,|\cdot|)$ to itself continuous is equivalent to $\|\cdot\|$.
\end{abstract}

\section{IntRodUCtion AND STATEMENTS OF THE THEOREMS}

Let $(A,\|\cdot\|)$ be a commutative complex Banach algebra and let $\Phi_{A}$ denote the set of all nonzero multiplicative linear functionals on $A$. It is a basic fact that these functionals are continuous. The carrier space of $A$ is the set $\Phi_{A}$ endowed with the Gelfand topology, which is the relative weak-star topology inherited from the topological dual space $A^{\prime}$ of $A . \Phi_{A}$ is a locally compact Hausdorff space and the Gelfand representation of $A$ is the mapping $a \mapsto \hat{a}$ from $A$ to the Banach algebra $C_{0}\left(\Phi_{A}\right)$ defined by $\hat{a}(\phi)=\phi(a)$. The hull-kernel topology on $\Phi_{A}$ is the topology on $\Phi_{A}$ whose closed sets are of the form $h(S)=\left\{\phi \in \Phi_{A}: \phi(S)=0\right\}$ with some $S \subset A$. This is a $T_{1}$-topology which is not necessarily Hausdorff and it is weaker than the Gelfand topology. In general the Gelfand topology and the hull-kernel topology are different and $A$ is regular if both of them coincide. For further information, we refer to [1, Chapters 17 and 23] and [4, Chapter 3].

From the uniform boundedness theorem and the continuity of multiplicative linear functionals on a Banach algebra, it follows immediately that any complete norm on the underlying linear space of a semisimple commutative Banach algebra $(A,\|\cdot\|)$ under which all the multiplication operators are continuous is automatically equivalent to $\|\cdot\|$. It is thus natural to ask whether or not the norm topology in the Banach algebra $A$ may be determined by a given multiplication operator by itself. An element $a$ in a commutative Banach algebra $(A,\|\cdot\|)$ is said to determine the norm topology of $A$ if every norm $|\cdot|$ on $A$ for which it becomes a Banach space and which makes the multiplication operator by $a$ from $(A,|\cdot|)$ to itself continuous is equivalent to $\|\cdot\|$. The element $a$ is said to almost determine the norm topology of $A$ if the following property holds: for every complete norm on $A$ making the multiplication by $a$ from $(A,|\cdot|)$ to itself continuous there exists an idempotent $e \in A$ such that $\operatorname{dim} e A<\infty$ and the quotient norms of $|\cdot|$ and $\|\cdot\|$ on $A /(e A)$ are equivalent.

Received by the editors May 19, 1999 and, in revised form, June 28, 1999.

2000 Mathematics Subject Classification. Primary 43A20, 46E25, 46J10, 46 J15.

(C)2000 American Mathematical Society 
The radical of $A$ is the subset of $A$ given by $\operatorname{Rad}(A)=\bigcap_{\phi \in \Phi_{A}} \operatorname{ker} \phi$ provided that $\Phi_{A} \neq \varnothing$ and $\operatorname{Rad}(A)=A$ otherwise. $A$ is semisimple if $\operatorname{Rad}(A)=0$ and it is radical if $\operatorname{Rad}(A)=A$.

In the present paper we prove the following two theorems.

Theorem 1. Let $A$ be a semisimple commutative complex Banach algebra and let $a \in A$.

(i) If, for every $\lambda \in \mathbb{C}$, the set $\left\{\phi \in \Phi_{A}: \hat{a}(\phi)=\lambda\right\}$ has no interior points for the hull-kernel topology on $\Phi_{A}$, then a determines the norm topology of $A$.

(ii) If, for every $\lambda \in \mathbb{C}$, the set $\left\{\phi \in \Phi_{A}: \hat{a}(\phi)=\lambda\right\}$ has a finite number of interior points for the hull-kernel topology on $\Phi_{A}$, then a almost determines the norm topology of $A$.

(iii) If there is $\lambda \in \mathbb{C}$ such that the set $\left\{\phi \in \Phi_{A}: \hat{a}(\phi)=\lambda\right\}$ has infinitely many interior points for the hull-kernel topology on $\Phi_{A}$, then a does not determine the norm topology of $A$.

Theorem 2. Let $A$ be a commutative complex Banach algebra and let $a \in A$ such that $\bigcap_{n=1}^{\infty} a^{n} \operatorname{Rad}(A)=0$. Assume that $A$ is an integral domain and that $\operatorname{dim} A=$ $\infty$. Then a determines the norm topology of $A$ if and only if it is not a complex multiple of the identity.

Remarks 1. 1. Since the hull-kernel topology on $\Phi_{A}$ is weaker than the Gelfand topology, assertions (i) and (ii) of Theorem \still hold if we replace the hull-kernel topology by the Gelfand topology. If $A$ is regular, then these topologies are the same.

2. The Banach algebras under consideration need not have an identity. For a Banach algebra without identity the assertion of $a \notin \mathbb{C} 1$ in Theorem 2 means that $a \neq 0$.

\section{EXAMPLES}

It should be noted that the class of semisimple commutative Banach algebras coincides with the class of Banach function algebras (regarded as being defined on the carrier space). We consider in the next corollary the suggestive form taken by Theorem 1 for regular Banach function algebras. For a locally compact Hausdorff space $\Omega$, let $C_{0}(\Omega)$ denote the space of continuous functions on $\Omega$ which vanish at infinity. With pointwise operations and the supremum norm $\|\cdot\|_{\infty}, C_{0}(\Omega)$ is a semisimple regular commutative Banach algebra. The nonzero multiplicative linear functionals on $C_{0}(\Omega)$ are the evaluation maps $\varepsilon_{\omega}(\omega \in \Omega)$ and the map $\omega \mapsto \varepsilon_{\omega}$ is a homeomorphism from $\Omega$ onto the carrier space. A subalgebra $A$ of $C_{0}(\Omega)$ is a natural Banach function algebra on $\Omega$ if it has the following properties: (i) $A$ separates the points of $\Omega$ and vanishes nowhere on $\Omega$, (ii) $A$ is a Banach algebra with respect to some norm, and (iii) the natural embedding $\omega \mapsto \varepsilon_{\omega}$ from $\Omega$ into $\Phi_{A}$ is onto.

Corollary. Let $A$ be a natural regular Banach function algebra on a locally compact Hausdorff space $\Omega$ and let $f \in A$.

(i) If $f$ is constant on no open subset of $\Omega$, then $f$ determines the norm topology of $A$.

(ii) If, for every $\lambda \in \mathbb{C}, f^{-1}(\lambda)$ has a finite number of interior points, then a almost determines the norm topology of $A$. 
(iii) If $f$ is constant on an infinite open subset of $\Omega$, then $f$ does not determine the norm topology of $C_{0}(\Omega)$.

Remarks 2. 1. Of course, if the topological space $\Omega$ has no isolated points, then the second assertion of the preceding result disappears and therefore $f$ determines the norm topology of $A$ if and only if it is constant on no open subset of $\Omega$. This fact was proved in [7] for the algebra $C(K)$ for a compact Hausdorff space $K$ without isolated points.

2. Let $A$ be as in the Corollary and let $e$ be an idempotent in $A$ such that $\operatorname{dim} e A<\infty$. Then there are $\omega_{1}, \ldots, \omega_{m}$ isolated points of $\Omega$ such that $e$ is the characteristic function of the set $\left\{\omega_{1}, \ldots, \omega_{m}\right\}$. Writing $E=\Omega \backslash\left\{\omega_{1}, \ldots, \omega_{m}\right\}$ we can assert that the quotient algebra $A /(e A)$ is isomorphic to the restriction algebra $A_{E}=\left\{f_{\mid E}: f \in A\right\}$.

Examples 1. 1. Of course, for a locally compact Hausdorff space $\Omega, C_{0}(\Omega)$ is a natural regular Banach function algebra on $\Omega . C^{n}([0,1]), B V C([0,1])$, and $A C([0,1])$ are natural regular Banach function algebras on $[0,1]$ (see [4, A.2.4/5]) and a function in any of these algebras determines the norm topology of the algebra if and only if it is constant on no open interval of $[0,1]$. For a compact metric space $K$ and $0<\alpha \leq 1, \operatorname{Lip}_{\alpha}(K)$ is a natural regular Banach function algebra on $K$ ([5] Section 2]).

2. If $G$ is a locally compact abelian group, then the group algebra $L^{1}(G)$ is a semisimple regular commutative Banach algebra. The carrier space of $L^{1}(G)$ may be identified, via the Fourier transform, with the dual group of $G$ and the Gelfand and Fourier transforms for $L^{1}(G)$ coincide. Consequently, Theorem 1 is true if we replace the Gelfand transform of $f \in L^{1}(G)$ and the carrier space of $L^{1}(G)$ by the Fourier transform of $f$ and the dual group of $G$, respectively. Since the dual group of $\mathbb{R}^{n}$ has no isolated points we conclude that $f \in L^{1}\left(\mathbb{R}^{n}\right)$ determines the norm topology of $L^{1}\left(\mathbb{R}^{n}\right)$ if and only if its Fourier transform is constant on no open subset of $\mathbb{R}^{n}$. For $\rho>0$ let $\chi_{[-\rho, \rho]}$ be the characteristic function of the interval $[-\rho, \rho]$. The Fourier transform of $\chi_{[-\rho, \rho]}$ is the function $\frac{\sin (2 \pi \rho x)}{\pi x}$ and therefore $\chi_{[-\rho, \rho]}$ determines the norm topology of $L^{1}(\mathbb{R})$.

In the next example we show that in the presence of isolated points in the carrier space even the most natural elements of the algebra can fail to determine the norm topology.

Example 2. Let $A=C([0,1] \cup\{2\})$ and consider the elements $a$ and $u$ in $A$ given by

$$
a(t)=\left\{\begin{array}{ll}
t & \text { if } t \in[0,1], \\
0 & \text { if } t=2,
\end{array} \quad \text { and } \quad u(t)= \begin{cases}0 & \text { if } t \in[0,1] \\
1 & \text { if } t=2 .\end{cases}\right.
$$

We claim that $\overline{a A} \neq a A$. By the Weierstrass approximation theorem, the function

$$
b(t)= \begin{cases}\sqrt{t} & \text { if } t \in[0,1] \\ 0 & \text { if } t=2\end{cases}
$$

lies in $\overline{a A}$. If there existed $c \in A$ such that $a(t) c(t)=b(t)$ for all $t \in[0,1]$, we would have $c(t)=t^{-1 / 2}$ for all $\left.\left.t \in\right] 0,1\right]$, a contradiction. Since $\overline{a A} \neq a A$ and $u \notin a A$, it follows that there exists a discontinuous linear functional $f$ on $C([0,1] \cup\{2\})$ such that $f(a A)=0$ and $f(u)=1$. The map $|b|=\|2 b-f(b) u\|$ defines a norm on $A$ for 
which it becomes a Banach space and $|\cdot|$ is not equivalent to $\|\cdot\|$. On the other hand,

$$
\begin{aligned}
|a b| & =\|2 a b-f(a b) u\|=\|2 a b\|=\|a(2 b-f(b) u)\| \\
& \leq\|a\|\|2 b-f(b) u\|=\|a\||b|
\end{aligned}
$$

for all $b \in A$, which shows that the multiplication by $a$ from $(A,|\cdot|)$ to itself is continuous.

Examples 3. 1. Let $\mathbb{C}[[x]]$ denote the algebra of formal power series with complex coefficients in the indeterminate $x$. A Banach algebra of power series is a Banach space $A$ which is a subalgebra of $\mathbb{C}[[x]]$ containing the indeterminate $x$ and such that the coefficient functionals are continuous. The closed graph theorem shows that multiplication is separately continuous, and so $A$ becomes a Banach algebra for an equivalent norm. Further $A$ is an integral domain and for any nonconstant power series $a$ in $A$ it is clear that $\bigcap_{n=1}^{\infty} a^{n} A=0$. Consequently, Theorem 2 shows that any nonconstant element of $A$ determines the norm topology of $A$. For an example of Banach algebra of power series, let $\omega$ be an algebra weight on $\mathbb{N}_{0}$, that is, $\omega$ is a function from $\mathbb{N}_{0}$ to $\mathbb{R}^{+}$satisfying $\omega(0)=1$ and $\omega(m+n) \leq \omega(m) \omega(n) \forall m, n \in \mathbb{N}$. Let

$$
\ell^{1}(\omega)=\left\{f=\sum_{n=0}^{\infty} \alpha_{n} x^{n}:\|f\|=\sum_{n=0}^{\infty}\left|\alpha_{n}\right| \omega(n)<\infty\right\} .
$$

$\ell^{1}(\omega)$ is a Banach algebra of power series. It is well known that $\ell^{1}(\omega)$ is semisimple if $\lim (\omega(n))^{1 / n}>0$ and $\operatorname{Rad}\left(\ell^{1}(\omega)\right)=\left\{f \in \ell^{1}(\omega): f(0)=0\right\}$ if $\lim (\omega(n))^{1 / n}=0$. For further information we refer the reader to [2].

2. Let $\omega$ be a positive measurable function on $[0,+\infty[\operatorname{such}$ that $\omega(s+t) \leq$ $\omega(s) \omega(t)$ for all $s, t \in[0,+\infty[$; such a function is said to be a weight function on $\left[0,+\infty\left[\right.\right.$. Let $L^{1}(\omega)$ denote the space of (equivalence classes of) measurable functions $f$ on $\left[0,+\infty\left[\right.\right.$ for which $\|f\|_{\omega}=\int_{0}^{\infty}|f(t)| \omega(t) d t<\infty . L^{1}(\omega)$ becomes a complex Banach algebra for the norm $\|\cdot\|_{\omega}$ and the convolution $(f * g)(t)=\int_{0}^{t} f(t-s) g(s) d s$ as product. For a thorough treatment of these algebras we refer the reader to [3]. It is known that $L^{1}(\omega)$ is an integral domain. It is semisimple if $\lim _{t \rightarrow \infty} \omega(t)^{1 / t}>0$, and radical if $\lim _{t \rightarrow \infty} \omega(t)^{1 / t}=0$. For $f \in L^{1}(\omega) \backslash\{0\}$, let

$$
\alpha(f)=\sup \{\delta \geq 0: f=0 \text { almost everywhere on }[0, \delta]\},
$$

and set $\alpha(0)=\infty$. If $f \in L^{1}(\omega)$ satisfies $\alpha(f) \neq 0$, then Titchmarsh's convolution theorem [3, Theorem 3.10] shows that $\bigcap_{n=1}^{\infty} f^{n} * L^{1}(\omega)=0$. According to Theorem2 we deduce that a nonzero function $f \in L^{1}(\omega)$ determines the norm topology of $L^{1}(\omega)$ provided that either $\lim _{t \rightarrow \infty} \omega(t)^{1 / t}>0$ or $\alpha(f) \neq 0$. Therefore for any weight function $\omega$ and $0<\rho_{1}<\rho_{2}<\infty$ the function $\chi_{\left[\rho_{1}, \rho_{2}\right]}$ determines the norm topology of $L^{1}(\omega)$.

\section{Proofs of the theorems}

Although the Banach algebras under consideration need not have an identity, given $a$ and $b$ in the algebra $A$ and $\lambda \in \mathbb{C}$, we write $(a+\lambda) b$ instead of $a b+\lambda b$.

If $X$ is a linear space, then we measure the equivalence of two norms $|\cdot|$ and $\|\cdot\|$ on $X$ for which $X$ becomes a Banach space by considering the separating subspace of the identity map from $(X,|\cdot|)$ onto $(X,\|\cdot\|)$. This is defined as the subspace $\mathfrak{S}$ of those $x \in X$ for which there exists a sequence $\left\{x_{n}\right\}$ in $X$ such that $\left\{x_{n}\right\}$ converges 
to zero for the norm $|\cdot|$ and converges to $x$ for the norm $\|\cdot\|$. The closed graph theorem shows that $|\cdot|$ and $\|\cdot\|$ are equivalent if, and only if, $\mathfrak{S}=0$.

Lemma 1. Let $X$ be a linear space and let $|\cdot|$ and $\|\cdot\|$ be two norms on $X$ for which it becomes a Banach space.

(i) If $F_{n}$ is a sequence of linear operators from $X$ to itself which are $|\cdot|-|\cdot|-$ continuous and $\|\cdot\|-\|\cdot\|$-continuous, then there exists $N \in \mathbb{N}$ such that ${\overline{F_{1} \cdots F_{N}(\mathfrak{S})}}^{\|\cdot\|}=\overline{F_{1} \cdots F_{n}(\mathfrak{S})}\|\cdot\|$ for all $n \geq N$.

(ii) If $F$ is a linear operator form $X$ to itself which is $|\cdot|-|\cdot|$-continuous and $\|\cdot\|-\|\cdot\|$ continuous, then there exists $N \in \mathbb{N}$ such that $\overline{F^{N}(\mathfrak{S})} \|^{\|\cdot\|}={\overline{\bigcap_{n=1}^{\infty} F^{n}(\mathfrak{S})}\|\cdot\|}$.

Proof. The first assertion follows immediately from [6, Lemma 1.6].

From (i) we see that there exists $N \in \mathbb{N}$ such that $\overline{F^{n}(\mathfrak{S})}\|\cdot\|={\overline{F^{N}(\mathfrak{S})}}^{\|\cdot\|}$ for all $n \geq N$. Therefore $F$ is a continuous linear operator from the Banach space $\left(\overline{F^{N}(\mathfrak{S})}\|\cdot\|,\|\cdot\|\right)$ onto a dense linear subspace of itself. According to Mittag-Leffler theorem [2, Theorem 5.3], we have

$$
{\overline{F^{N}(\mathfrak{S})}}^{\|\cdot\|}=\bar{\bigcap}_{n=1}^{\infty} F^{n}\left({\overline{F^{N}(\mathfrak{S})}}^{\|\cdot\|}\right)\|\cdot\|
$$

It is immediate that $F(\mathfrak{S}) \subset \mathfrak{S}$ and that $\mathfrak{S}$ is closed in $(X,\|\cdot\|)$, and so $\overline{F^{N}(\mathfrak{S})}\|\cdot\|$ S. Hence $\bigcap_{n=1}^{\infty} F^{n}\left(\overline{F^{N}(\mathfrak{S})}\|\cdot\|\right) \subset \bigcap_{n=1}^{\infty} F^{n}(\mathfrak{S}) \subset \overline{F^{B}(\mathfrak{S})}\|\cdot\|$, and our assertion follows.

Lemma 2. Let $(A,\|\cdot\|)$ be a commutative complex Banach algebra and let $a \in A$. Assume that $|\cdot|$ is a norm on A for which $A$ is a Banach space and the multiplication operator by a from $(A,|\cdot|)$ to itself is continuous and let $\mathfrak{S}$ be the separating subspace of the identity map from $(A,|\cdot|)$ onto $(A,\|\cdot\|)$. Then there are $\lambda_{1}, \ldots, \lambda_{m} \in \mathbb{C}$ such that $\left(a-\lambda_{1}\right) \cdots\left(a-\lambda_{m}\right) \mathfrak{S} \subset \operatorname{Rad}(A)$.

Proof. We claim that the set $\left\{\phi(a): \phi \in \Phi_{A} \backslash h(\mathfrak{S})\right\}$ is finite, where $h(\mathfrak{S})=\{\phi \in$ $\left.\Phi_{A}: \phi(\mathfrak{S})=0\right\}$. Suppose the claim were false. Then we could find a sequence $\left\{\phi_{n}\right\}$ in $\Phi_{A} \backslash h(\mathfrak{S})$ such that $\phi_{n}(a) \neq \phi_{m}(a)$ if $n \neq m$. For every $n \in \mathbb{N}$ the linear operator $b \longmapsto\left(a-\phi_{n}(a)\right) b$ from $A$ to itself is $|\cdot|-|\cdot|$-continuous and $\|\cdot\|-\|\cdot\|$-continuous. From Lemma 1)(i) we deduce that there exists $N \in \mathbb{N}$ such that

$$
{\overline{\left(a-\phi_{1}(a)\right) \cdots\left(a-\phi_{N}(a)\right) \mathfrak{S}}}^{\|\cdot\|}={\overline{\left(a-\phi_{1}(a)\right) \cdots\left(a-\phi_{n}(a)\right) \mathfrak{S}}}^{\|\cdot\|}
$$

for all $n \geq N$. Consequently,

$$
\left(a-\phi_{1}(a)\right) \cdots\left(a-\phi_{N}(a)\right) \mathfrak{S} \subset \overline{\left(a-\phi_{1}(a)\right) \cdots\left(a-\phi_{N+1}(a)\right) \mathfrak{S}}{ }^{\|\cdot\|} .
$$

Since $\phi_{N+1}$ is continuous on $(A,\|\cdot\|)$, we see that

$$
\begin{aligned}
\left(\phi_{N+1}\right. & \left.(a)-\phi_{1}(a)\right) \cdots\left(\phi_{N+1}(a)-\phi_{N}(a)\right) \phi_{N+1}(\mathfrak{S}) \\
& =\phi_{N+1}\left(\left(a-\phi_{1}(a)\right) \cdots\left(a-\phi_{N}(a)\right) \mathfrak{S}\right) \\
& \subset \overline{\phi_{N+1}\left(\left(a-\phi_{1}(a)\right) \cdots\left(a-\phi_{N+1}(a)\right) \mathfrak{S}\right)}=0
\end{aligned}
$$

which clearly forces $\phi_{N+1}(\mathfrak{S})=0$, a contradiction. Let $\phi_{1}, \ldots, \phi_{m} \in \Phi_{A} \backslash h(\mathfrak{S})$ be such that $\left\{\phi(a): \phi \in \Phi_{A} \backslash h(\mathfrak{S})\right\}=\left\{\phi_{1}(a), \ldots, \phi_{m}(a)\right\}$. We proceed to show that $\phi\left(\left(a-\phi_{1}(a)\right) \cdots\left(a-\phi_{m}(a)\right) \mathfrak{S}\right)=0$ for all $\phi \in \Phi_{A}$. This equality is easily seen to be 
true whenever $\phi$ lies in $h(\mathfrak{S})$. On the other hand, if $\phi \notin h(\mathfrak{S})$, then $\phi(a)=\phi_{k}(a)$ for some $k \in\{1, \ldots, m\}$ and therefore the equality holds.

Lemma 3. Let $X$ be a topological space, let $C_{1}, \ldots, C_{n}$ be closed subsets of $X$, and let $D$ be an open subset of $X$ such that $D \subset \bigcup_{k=1}^{n} C_{k}$. Assume that, for each $k \in\{1, \ldots, n\}$, the interior $C_{k}^{\circ}$ of $C_{k}$ is closed. Then $D \subset \bigcup_{k=1}^{n} C_{k}^{\circ}$.

Proof of Theorem [1] Let $\|\cdot\|$ be the norm of $A$ and let $|\cdot|$ be a norm on $A$ for which $A$ is a Banach space and the multiplication operator by $a$ from $(A,|\cdot|)$ to itself is continuous. Let $\mathfrak{S}$ be the separating subspace of the identity map from $(A,|\cdot|)$ onto $(A,\|\cdot\|)$. Lemma 2 gives $\lambda_{1}, \ldots, \lambda_{m} \in \mathbb{C}$ such that $\left(a-\lambda_{1}\right) \cdots\left(a-\lambda_{m}\right) \mathfrak{S}=0$. Consequently, $\Phi_{A} \backslash h(\mathfrak{S}) \subset \bigcup_{k=1}^{m}\left\{\phi \in \Phi_{A}: \phi(a)=\lambda_{k}\right\}$. Let us suppose that, for every $\lambda \in \mathbb{C}$, the set $\left\{\phi \in \Phi_{A}: \hat{a}(\phi)=\lambda\right\}$ has a finite number of interior points for the hull-kernel topology on $\Phi_{A}$ and let us consider the topological space $X=\Phi_{A}$ endowed with the hull-kernel topology. For each $k \in\{1, \ldots, m\}$ the set $C_{k}=\{\phi \in$ $\left.\Phi_{A}: \phi(a)=\lambda_{k}\right\}$ is closed in $X\left(\left[4\right.\right.$, Lemma 3.1.16]) and its interior $C_{k}^{\circ}$ is finite and so it is closed. Lemma 3 now yields $\Phi_{A} \backslash h(\mathfrak{S}) \subset \bigcup_{k=1}^{m} C_{k}^{\circ}$. Consequently, if the requirement in the first assertion is fulfilled, then we can assert that $\Phi_{A} \backslash h(\mathfrak{S})=$ $\varnothing$. Thus $\phi_{A} \backslash h(\mathfrak{S})=\varnothing$. Thus $\phi(\mathfrak{S})=0$ for all $\phi \in \Phi_{A}$ and therefore $\mathfrak{S}=0$, which shows that $|\cdot|$ and $\|\cdot\|$ are equivalent. We now turn to the case traced in our second assertion. From what has already been proved, it may be concluded that $\Phi_{A} \backslash h(\mathfrak{S})=\left\{\phi_{1}, \ldots, \phi_{m}\right\}$ with $\phi_{1}, \ldots, \phi_{m} \in \Phi_{A}$ and note that, for each $k \in$ $\{1, \ldots, m\}$, the set $\left\{\phi_{k}\right\}$ is open. Let $k \in\{1, \ldots, m\}$. Since $\Phi_{A} \backslash\left\{\phi_{k}\right\}$ is closed and $\phi_{k} \notin \Phi_{A} \backslash\left\{\phi_{k}\right\}$, [1, Proposition 23.4] gives $e_{k} \in A$ such that $\phi_{k}\left(e_{k}\right)=1$ and $\phi\left(e_{k}\right)=0$ for all $\phi \neq \phi_{k}$. It is immediate that $\phi\left(e_{k}^{2}-e_{k}\right)=0$ for all $\phi \in \Phi_{A}$, and so $e_{k}^{2}=e_{k}$. Furthermore, if $i, j \in\{1, \ldots, m\}$ with $i \neq j$, then $\phi\left(e_{i} e_{j}\right)=0$ for all $\phi \in \Phi_{A}$, which implies $e_{i} e_{j}=0$. Thus $e=e_{1}+\cdots+e_{m}$ is an idempotent, $\phi_{k}(e)=1$ for $k=1, \ldots, m$, and $\phi(e)=0$ for all $\phi \in \Phi_{A} \backslash\left\{\phi_{1}, \ldots, \phi_{m}\right\}$. The map $e b \mapsto\left(\phi_{1}(b), \ldots, \phi_{m}(b)\right)$ from $e A$ to $\mathbb{C}^{m}$ is easily seen to be injective and therefore $\operatorname{dim} e A<\infty$. It follows immediately that $\phi((1-e) \mathfrak{S})=0$ for all $\phi \in \Phi_{A}$ and therefore $(1-e) \mathfrak{S}=0$. The closed graph theorem now shows that the map $b \mapsto b-e b$ from $(A,|\cdot|)$ to $(A,\|\cdot\|)$ is continuous. Let $\left\{a_{n}\right\}$ be a sequence in $A$ such that $\left\{a_{n}+e A\right\}$ converges to zero in the quotient Banach space $(A /(e A),|\cdot|)$. Then there exists a sequence $\left\{b_{n}\right\}$ in $A$ such that $\left\{a_{n}+e b_{n}\right\}$ converges to zero in $(A,|\cdot|)$ and hence $a_{n}-e a_{n}=(1-e)\left(a_{n}+e b_{n}\right)$ converges to zero in $(A,\|\cdot\|)$, which shows that the sequence $\left\{a_{n}+e A\right\}$ converges to zero in the quotient Banach space $(A /(e A),\|\cdot\|)$. From this we conclude that the quotient norms of $|\cdot|$ and $\|\cdot\|$ on $A /(e A)$ are equivalent.

We finally assume that the set $\left\{\phi \in \Phi_{A}: \hat{a}(\phi)=\lambda\right\}$ has an infinitely many interior, say $D$, for the hull-kernel topology and some $\lambda \in \mathbb{C}$. Let $\left\{\phi_{n}\right\}$ be a sequence of pairwise different elements of $D$. Since $\Phi_{A} \backslash D$ is closed and $\phi_{1} \notin \Phi_{A} \backslash D$, 1. Proposition 23.4(i)] gives $a_{1} \in A$ such that $\phi_{1}\left(a_{1}\right)=1$ and $\phi\left(a_{1}\right)=0$ for all $\phi \in \Phi_{A} \backslash D$. For each $n \in \mathbb{N}$ we can choose $a_{n+1} \in A$ such that $\phi_{k}\left(a_{n+1}\right)=0$ for $k=1, \ldots, n, \phi_{n+1}\left(a_{n+1}\right) \neq 0$, and $\left\|a_{n+1}\right\|=1$ (see [1, Lemma 18.17]). It is a simple matter to show that the set $\left\{a_{n}: n \in \mathbb{N}\right\}$ is linearly independent and the intersection of $\bigcap_{\phi \in D} \operatorname{ker} \phi$ with the linear subspace spanned by $\left\{a_{n}: n \in \mathbb{N}\right\}$ is zero. Consequently, there is a linear functional $f$ on $A$ such that $f(b)=0$ for all $b \in \bigcap_{\phi \in D}$ ker $\phi$ and $f\left(a_{n}\right)=n$ for all $n \in \mathbb{N}$. Of course $f$ is discontinuous and therefore the map $|b|=\left\|2 b-f(b) a_{1}\right\|$ defines a norm on $A$ for which it becomes a Banach space and $|\cdot|$ is not equivalent to $\|\cdot\|$. The proof is completed by showing 
that the multiplication operator by $a$ from $(A,|\cdot|)$ to itself is continuous. For every $b \in A$, we see at once that $(a-\lambda) b \in \bigcap_{\phi \in D} \operatorname{ker} \phi$ and therefore we have $0=f((a-\lambda) b)=f(a b)-\lambda f(b)$. Since $\phi(a)=\lambda$ for all $\phi \in D$ and $\phi\left(a_{1}\right)=0$ for all $\phi \in \Phi_{A} \backslash D$, we conclude that $\phi\left((a-\lambda) a_{1}\right)=0$ for all $\phi \in \Phi_{A}$ and hence that $a a_{1}=\lambda a_{1}$. Consequently,

$$
\begin{aligned}
|a b| & =\left\|2 a b-f(a b) a_{1}\right\|=\left\|2 a b-\lambda f(b) a_{1}\right\| \\
& =\left\|2 a b-f(b) a a_{1}\right\| \leq\|a\|\left\|2 b-f(b) a_{1}\right\|=\|a\||| b \mid
\end{aligned}
$$

for all $b \in A$, which establishes the desired conclusion.

Proof of Theorem 娄 If $a=\lambda 1$ for some $\lambda \in \mathbb{C}$, then for any discontinuous linear functional $f$ on $A$ and any element $u \in A$ such that $f(u)=1$ the map $|b|=$ $\|2 b-f(b) u\|$ defines a complete norm on $A$ which clearly makes the multiplication by $a$ continuous and it is not equivalent to the norm of $A$.

Assume that $a$ is not a complex multiple of the identity. Let $\|\cdot\|$ be the norm of $A$ and let $|\cdot|$ be a norm on $A$ for which $A$ is a Banach space and the multiplication operator by $a$ from $(A,|\cdot|)$ to itself is continuous. Let $\mathfrak{S}$ be the separating subspace of the identity map from $(A,|\cdot|)$ onto $(A,\|\cdot\|)$. By considering the complex numbers $\lambda_{1}, \ldots, \lambda_{m}$ given in Lemma 2 we have

$$
\left(a-\lambda_{1}\right) \cdots\left(a-\lambda_{m}\right) \mathfrak{S} \subset \operatorname{Rad}(A) .
$$

From Lemma 1(ii) it follows that there exists $N \in \mathbb{N}$ such that

$$
\overline{a^{N}\left(a-\lambda_{1}\right)^{N} \cdots\left(a-\lambda_{m}\right)^{N} \mathfrak{S}}\|\cdot\|=\bigcap_{n=1}^{\infty}\left[a\left(a-\lambda_{1}\right) \cdots\left(a-\lambda_{m}\right)\right]^{n} \mathfrak{S},
$$

which gives

$$
\begin{aligned}
a^{N}\left(a-\lambda_{1}\right)^{N} \cdots\left(a-\lambda_{m}\right)^{N} \mathfrak{S} & \subset \bigcap_{n=1}^{\infty} a^{n}\left[\left(a-\lambda_{1}\right) \cdots\left(a-\lambda_{m}\right)\right]^{n} \mathfrak{S} \\
& \subset \bigcap_{n=1}^{\infty} a^{n} \operatorname{Rad}(a)=0 .
\end{aligned}
$$

We claim that $\mathfrak{S}=0$. On the contrary, suppose that $\mathfrak{S} \neq 0$. Since $A$ is an integral domain, it follows that $\left[a\left(a-\lambda_{1}\right) \cdots\left(a-\lambda_{m}\right)\right]^{n}=0$ and therefore $a\left(a-\lambda_{1}\right) \cdots\left(a-\lambda_{m}\right)=0$. Since $a\left[\left(a-\lambda_{1}\right) A\right] \cdots\left[\left(a-\lambda_{m}\right) A\right]=0$ it may be concluded that $\left(a-\lambda_{k}\right) A=0$ for some $k \in\{1, \ldots, m\}$. Clearly $A_{k} \neq 0$, and hence $\left(\lambda_{k}^{-1} a\right) b=b$ for all $b \in A$, a contradiction.

\section{REFERENCES}

1. F. F. Bonsall and J. Duncan, Complete normed algebras, Springer-Verlag 1973. MR 54:11013

2. H. G. Dales, Automatic continuity: a survey, Bull. London Math. Soc. 10 (1978), 129-183. MR 80c:46053

3. H. G. Dales, Convolution algebras on the real line. In Radical Banach algebras and automatic continuity, Springer Lecture Notes in Math. 975, 180-209 (1983). MR 84c:46001

4. C. E. Rickart, General theory of Banach algebras, Robert E. Krieger Publishing Co. 1974. MR 22:5903 (review of original 1960 edition)

5. D. R. Sherbert, The structure of ideals and point derivations in Banach algebras of Lipschitz functions, Trans. Amer. Math. Soc. 111 (1964), 240-272. MR 28:4385 
6. A. M. Sinclair, Automatic continuity of linear operators, Cambridge University Press, 1976. MR 58:7011

7. A. R. Villena, Operators determining the complete norm topology of $C(K)$, Studia Math. 124 (1997), 155-160. MR 98h:46026

Departamento de Analisis Matematico, Facultad de Ciencias, Universidad de Granada, 18071 Granada, Spain

E-mail address: avillena@ugr.es 